\title{
Updating the Free Radical Theory of Aging
}

\author{
Adam S. Ziada ${ }^{1,2}$, Marie-Soleil R. Smith ${ }^{1,2}$ and Hélène C. F. Côté ${ }^{1,2,3 *}$ \\ ${ }^{1}$ Department of Pathology and Laboratory Medicine, University of British Columbia, Vancouver, BC, Canada, ${ }^{2}$ Centre for \\ Blood Research, University of British Columbia, Vancouver, BC, Canada, ${ }^{3}$ Women's Health Research Institute, Vancouver, \\ $B C$, Canada
}

Keywords: free radical theory of aging, mtDNA, mtDNA mutations, oxidative stress, clonal expansion, polymerase gamma (POLG), mtDNA replication, aging

\section{INTRODUCTION}

OPEN ACCESS

Edited by:

Miguel A. Aon,

National Institute on Aging $(\mathrm{NIH})$

United States

Reviewed by:

Plácido Navas,

Universidad Pablo de Olavide, Spain

*Correspondence:

Hélène C. F. Côté

helene.cote@ubc.ca

Specialty section:

This article was submitted to

Mitochondrial Research,

a section of the journal

Frontiers in Cell and Developmental

Biology

Received: 23 June 2020

Accepted: 18 August 2020

Published: 16 September 2020

Citation:

Ziada AS, Smith MSR and Côté HCF (2020) Updating the Free Radical

Theory of Aging.

Front. Cell Dev. Biol. 8:575645.

doi: 10.3389/fcell.2020.575645
The free radical theory of aging, one of the nine suggested hallmarks of aging (López-Otín et al., 2016), implicates the gradual accumulation of oxidative cellular damage as a fundamental driver of cellular aging (Harman, 1956; Miquel et al., 1980). This theory has evolved over time to emphasize the role of free radical induced mitochondrial DNA (mtDNA) mutations and the accumulation of mtDNA deletions (Miquel et al., 1980; Cortopassi et al., 1992; Michikawa et al., 1999). Given the proximity of mtDNA to the electron transport chain, a primary producer of free radicals, it postulates that the mutations would promote mitochondrial dysfunction and concomitantly increase free radical production in a positive feedback loop. The observation of oxidative damage in the form of 7,8-dihydro-8-oxo-deoxyguanosine (8-oxodG) DNA oxidative lesions accumulating with age has been a cornerstone of the free radical theory of aging (Fraga et al., 1990).

\section{THE INFLUENCE OF STRESSORS ON MTDNA MUTATION BURDEN AND AGING}

A major assumption of the free radical theory of aging is that random de novo or somatic mtDNA mutations gradually accumulate over time, eventually reaching pathological levels (Harman, 1956, 1972). However, data from Payne et al. support the hypothesis that, rather than gradually accumulating over time, mtDNA turnover can lead to the clonal expansion of pre-existing age-related mutations (Payne et al., 2011). Once amplified, these higher frequency mtDNA mutations, that are potentially pathogenic, are referred to as heteroplasmy.

To further understand the potential link between mtDNA mutations and the free radical theory of aging, our group examined aging in the context of tobacco smoking and human immunodeficiency virus (HIV) infection, both believed to accelerate aging. While smoking has long been known for its association with accelerated aging and oxidative damage (Kiyosawa et al., 1990; Loft et al., 1992), HIV infection is also increasingly studied as a promoter of accelerated aging (Effros et al., 2008; Deeks and Phillips, 2009). HIV-positive individuals experience 
a reported decrease in lifespan of up to 10 years (Lohse et al., 2007; Antiretroviral Therapy Cohort Collaboration, 2008), as well as earlier onset and higher prevalence of agerelated comorbidities (Guaraldi et al., 2011). These include cardiovascular disease, hypertension, diabetes, bone disease, and renal failure among others, even in individuals whose viremia is controlled by antiretroviral therapy (Guaraldi et al., 2011).

Our study found that both random somatic and heteroplasmic mtDNA mutations, the latter defined as a frequency $>2 \%$, were associated with older age (Ziada et al., 2019). Further, our data suggest that smoking and HIV may distinctly contribute to the accumulation of mtDNA mutations. Indeed, smoking showed an association with increased mtDNA heteroplasmy but not somatic mutations, while the reverse was observed with HIV participants, but only in those with a history of high viremia, reflecting poor control of HIV. These results suggest that the chronic immune activation and subsequent oxidative stress induced by HIV may lead to de novo mtDNA mutations, while oxidative damage associated with exposure to tobacco smoking may promote the clonal amplification of pre-existing mtDNA mutations (Figure 1).

Consistent with this model, no heteroplasmic transversion mutations, typically a signature of oxidative damage, were observed among the 164 participants studied (Ziada et al., 2019). Furthermore, within a given participant showing heteroplasmy, identically mutated molecules of mtDNA would be repeatedly observed; however, between participants, the pattern of heteroplasmic mutation was generally unique (Ziada et al., 2019). Such a pattern is not consistent with the gradual build-up of random mtDNA mutations. Taken together, our findings do not support the slow accumulation of mtDNA transversion mutations as proposed by the free radical theory of aging. Rather, they suggest that randomly mutated molecules of mtDNA are being clonally amplified to generate unique patterns of heteroplasmy in our participants.

\section{MTDNA DAMAGE AND THE ROLE OF POLYMERASE $\gamma$}

Although the accumulation of mtDNA mutations has been linked to older age and age-associated conditions (Michikawa et al., 1999), several studies have provided new insight that challenge the connection between oxidative damage and mtDNA mutations. For example, the most studied oxidative lesion, 8oxodG (Yasui et al., 2014), is one of the 37 major oxidative lesions, and is known to induce transversion mutations $(\mathrm{A} \leftrightarrow \mathrm{C}, \mathrm{A} \leftrightarrow \mathrm{T}$, $\mathrm{C} \leftrightarrow \mathrm{G}, \mathrm{G} \leftrightarrow \mathrm{T}$ ) (Evans et al., 2004). However, recent studies showing the accumulation of mtDNA mutations with aging did not observe increases in mtDNA transversion mutations, but rather increases in mtDNA transition mutations $(A \leftrightarrow G, C \leftrightarrow$ T) (Trifunovic et al., 2004; Kennedy et al., 2013), believed to be the hallmark of mitochondrial polymerase $\gamma$ errors rather than oxidative damage (Spelbrink et al., 2000; Longley et al., 2001; Kauppila et al., 2017). Additionally, in our study, although both somatic transition and transversion mutations increased with older age, transition mutations were over 30 times more abundant than transversion mutations, once again suggesting that mtDNA replication errors are the major contributors to mtDNA mutation burden (Ziada et al., 2019).

Mutations in mitochondrial polymerase $\gamma$, responsible for mtDNA replication in mammalian cells (Hübscher et al., 1979), can be broadly pathogenic and reduce fidelity, resulting in mtDNA replication errors. Over time these errors, including mtDNA point mutations, may undergo clonal expansion, reaching pathogenic levels within the organisms lifetime. Among the first to highlight the importance of polymerase $\gamma$ errors was a study performed by Trifunovic et al. using mutator mice with proof-reading-deficient mitochondrial polymerase $\gamma$. These mice not only showed an increase in mtDNA mutation burden, the vast majority of which were transition mutations, they also displayed a reduced lifespan and the premature onset of agingrelated phenotype with no evidence of increased oxidative stress (Trifunovic et al., 2004). This paper provided a causative link between the buildup of polymerase induced mtDNA mutations and aging, forming the foundation for a new polymerase $\gamma$ focused theory of mitochondrial aging (Trifunovic et al., 2004; Matkarimov and Saparbaev, 2020).

The development of the polymerase $\gamma$ theory of aging led to renewed examination of the links between free radicals and mitochondrial aging. Mirroring the results in humans (Kennedy et al., 2013; Ziada et al., 2019), a recent study in Drosophila showed that age was associated with accumulation of somatic mtDNA transition, but not transversion, mutations suggesting that the role of polymerase $\gamma$ errors in mitochondrial aging is not limited to humans (Itsara et al., 2014). Building on these results, neither the loss-of-function of antioxidant or DNA damage repair enzymes were shown to increase somatic mtDNA point mutation burden in that model (Itsara et al., 2014). Taken together, these findings lend strong support to the role of polymerase $\gamma$ errors, with respect to their direct contribution toward somatic mtDNA point mutation burden, and confine free radicals to a comparatively minor role.

Understanding the role that free radicals may play in a polymerase $\gamma$ centric model of mitochondrial aging is an active area of research. Zsurka et al. suggest that oxidative stress may induce mtDNA deletions rather than point mutations, and that the polymerase $\gamma$ nucleotide selectivity may prevent the fixation of 8-oxodG induced transversion mutations (Zsurka et al., 2018). Nevertheless, while the 8-oxodG lesion may not directly induce lasting transversion mutations (Basu et al., 1989; Kreutzer and Essigmann, 1998), one or more of the other 37 products of oxidative damage (Evans et al., 2004), some of which induce transition mutations, may contribute to the somatic mtDNA transition mutation burden observed with older age (Kennedy et al., 2013; Ziada et al., 2019).

It has also been suggested that under specific circumstances, reactive oxygen species (ROS) may play an important role in normal mitochondrial function. One Drosophila study demonstrated that while ROS production increases with age and correlates with the functional deterioration of mitochondria, increasing ROS production at a choice site within the electron transport chain can act as a signal to maintain mitochondrial function and extend lifespan (Scialò et al., 2016). Studies in 


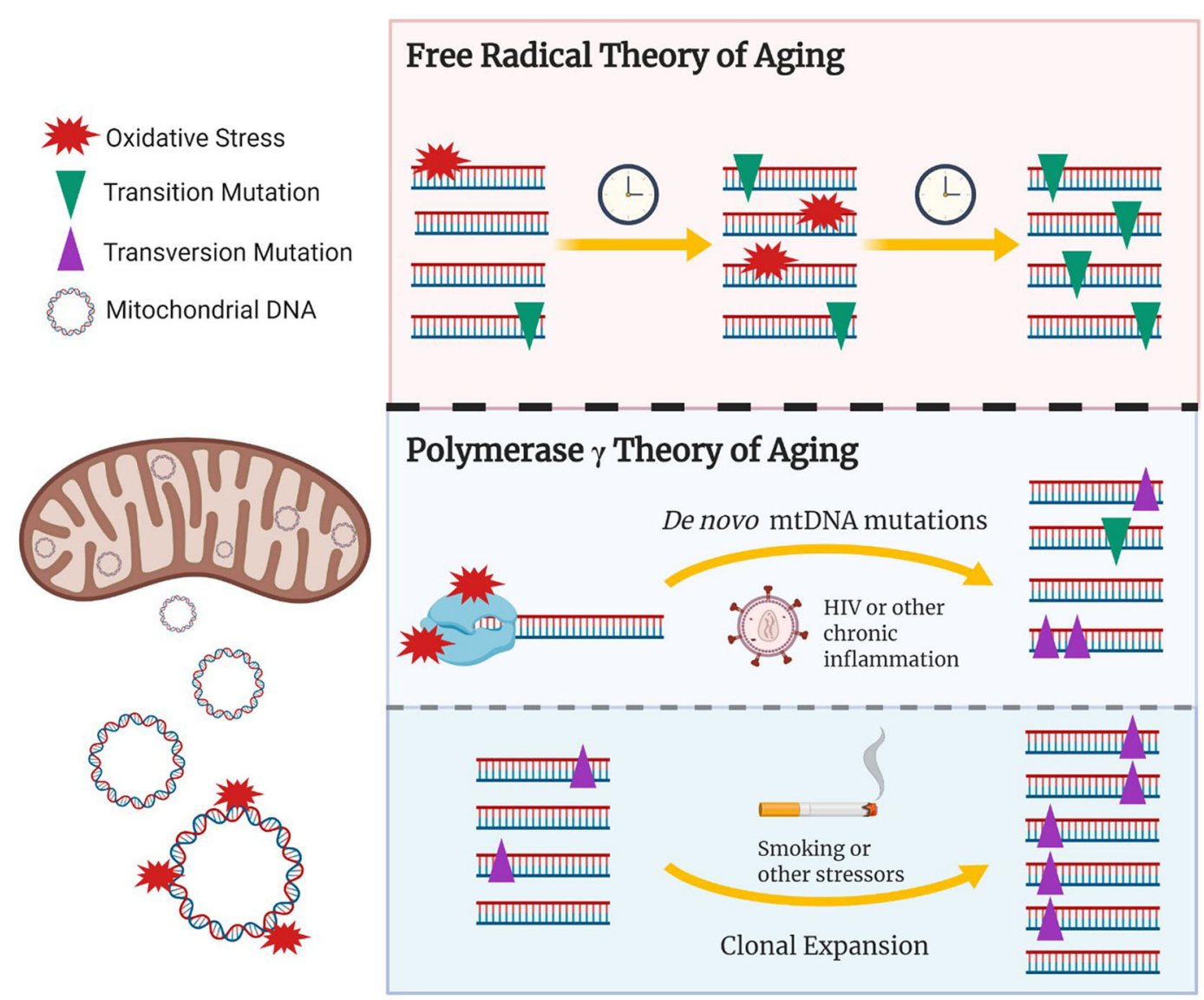

FIGURE 1 | The updated role of oxidative damage and mtDNA polymerase $\gamma$ in age-associated mtDNA mutations. The free radical theory of aging hypothesizes that oxidative damage to the mtDNA induces random de novo mtDNA mutations which gradually accumulate over time, potentially reaching pathological levels. Recent studies have shown that transition mtDNA mutations (purple triangles) rather than transversion mutations (green inverted triangles) gradually build up overtime and are amplified, via clonal expansion, to pathological levels. Given that transition mutations are generally associated with replication errors made by the mitochondrial polymerase $\gamma$, the age associated accumulation of mtDNA mutations could result from free radicals interacting with polymerase $\gamma$, potentially reducing its fidelity and/or inhibiting mtDNA replication. This would in turn lead to random de novo transition mutations and their subsequent clonal amplification. Conditions hypothesized to induce accelerated aging via oxidative damage/stress could include chronic infections such as HIV, chronic inflammatory conditions, or tobacco smoking. Stressors that induce mitochondrial biogenesis or cellular turnover, which could be mediated via oxidative stress, would in turn promote clonal expansion of existing damage. Created with BioRender.com.

C. elegans have also suggested that low levels of mitochondrial stress may be protective and extend longevity (Palikaras et al., 2015; Merkwirth et al., 2016). These findings fit into our growing understanding of the role ROS may play in mitochondrial aging, whereby low ROS levels they may be beneficial, but in excess contribute to mitochondrial dysfunction. Through their interaction with mitochondrial polymerase $\gamma$, excess of ROS could potentially reduce the enzyme's fidelity, indirectly contributing to an age-associated increase in somatic mtDNA mutations.

An alternate model proposed by Matkarimov and Saparbaev suggest that the spontaneous decay of mtDNA, rather than the accumulation of polymerase $\gamma$ errors, could be a major source of endogenous mutations (Matkarimov and Saparbaev, 2020). This model is predicated on the spontaneous decomposition of DNA bases (Lindahl and Andersson, 1972; Lindahl and
Karlstrom, 1973; Lindahl and Nyberg, 1974), a process that is accelerated when the DNA is in a single stranded form, such as during mtDNA replication, and would result in the accumulation of transition mutations over time (Matkarimov and Saparbaev, 2020). While this model very effectively explains the accumulation of somatic transversion mutations with older age (Kennedy et al., 2013; Ziada et al., 2019), mechanism(s) have been put forward by which such decay could be affected by factors and diseases with increased somatic transition mtDNA mutations (Ju et al., 2014; Ziada et al., 2019; Matkarimov and Saparbaev, 2020).

\section{CONCLUSION}

Recent research support the theory that mtDNA replication errors are the major drivers of cellular mtDNA mutation burden 
(Trifunovic et al., 2004; Kennedy et al., 2013; Kauppila et al., 2017). Nonetheless they do not exclude a comparatively minor role for 8-oxodG-induced transversion mutations, or the many other DNA oxidative lesions that can induce transition mutations (Basu et al., 1989; Kreutzer and Essigmann, 1998). Based on recent findings, an updated understanding regarding the role of free radicals in contemporary theories of mtDNA aging is needed. It seems likely that rather than directly contributing to mtDNA mutations via oxidative lesions, free radicals may affect the mitochondrial polymerase and decrease its fidelity, indirectly increasing somatic transition mutations. ROS may also act as a signaling molecule and influence mitochondrial biogenesis and/or mitochondrial turnover, which could in turn promote the clonal expansion of pre-existing mtDNA mutations.

\section{REFERENCES}

Antiretroviral Therapy Cohort Collaboration (2008). Life expectancy of individuals on combination antiretroviral therapy in high-income countries: a collaborative analysis of 14 cohort studies. Lancet 372, 293-299. doi: 10.1016/S0140-6736(08)61113-7

Basu, A. K., Loechler, E. L., Leadon, S. A., and Essigmann, J. M. (1989). Genetic effects of thymine glycol: site-specific mutagenesis and molecular modeling studies. Proc. Natl. Acad. Sci. U.S.A. 86, 7677-7681. doi: $10.1073 /$ pnas.86.20.7677

Cortopassi, G. A., Shibata, D., Soong, N. W., and Arnheim, N. (1992). A pattern of accumulation of a somatic deletion of mitochondrial DNA in aging human tissues. Proc. Natl. Acad. Sci. U.S.A. 89, 7370-7374. doi: $10.1073 /$ pnas.89.16.7370

Deeks, S. G., and Phillips, A. N. (2009). HIV infection, antiretroviral treatment, ageing, and non-AIDS related morbidity. BMJ 338:a3172. doi: 10.1136/bmj.a3172

Effros, R. B., Fletcher, C. V., Gebo, K., Halter, J. B., Hazzard, W. R., Horne, F. M., et al. (2008). Aging and infectious diseases: workshop on HIV infection and aging: what is known and future research directions. Clin. Infect. Dis. 47, 542-553. doi: 10.1086/590150

Evans, M. D., Dizdaroglu, M., and Cooke, M. S. (2004). Oxidative DNA damage and disease: induction, repair and significance. Mutat. Res. 567, 1-61. doi: 10.1016/j.mrrev.2003.11.001

Fraga, C. G., Shigenaga, M. K., Park, J. W., Degan, P., and Ames, B. N. (1990). Oxidative damage to DNA during aging: 8-hydroxy-2'-deoxyguanosine in rat organ DNA and urine. Proc. Natl. Acad. Sci. U.S.A. 87, 4533-4537. doi: $10.1073 /$ pnas.87.12.4533

Guaraldi, G., Orlando, G., Zona, S., Menozzi, M., Carli, F., Garlassi, E., et al. (2011). Premature age-related comorbidities among HIV-infected persons compared with the general population. Clin. Infect. Dis. 53, 1120-1126. doi: $10.1093 / \mathrm{cid} / \mathrm{cir} 627$

Harman, D. (1956). Aging: a theory based on free radical and radiation chemistry. J. Gerontol. 11, 298-300. doi: 10.1093/geronj/11.3.298

Harman, D. (1972). The biologic clock: the mitochondria? J. Am. Geriatr. Soc. 20, 145-147. doi: 10.1111/j.1532-5415.1972.tb00787.x

Hübscher, U., Kuenzle, C. C., and Spadari, S. (1979). Functional roles of DNA polymerases beta and gamma. Proc. Natl. Acad. Sci. U.S.A. 76, 2316-2320. doi: 10.1073/pnas.76.5.2316

Itsara, L. S., Kennedy, S. R., Fox, E. J., Yu, S., Hewitt, J. J., SanchezContreras, M., et al. (2014). Oxidative stress is not a major contributor to somatic mitochondrial DNA mutations. PLoS Genet. 10:e1003974. doi: 10.1371/journal.pgen.1003974

Ju, Y. S., Alexandrov, L. B., Gerstung, M., Martincorena, I., Nik-Zainal, S., Ramakrishna, M., et al. (2014). Origins and functional consequences of somatic mitochondrial DNA mutations in human cancer. Elife 3:e02935. doi: 10.7554/eLife.02935

\section{AUTHOR CONTRIBUTIONS}

AZ, M-SS, and HC conceived, designed, and wrote the manuscript. All authors contributed to the article and approved the submitted version.

\section{FUNDING}

This work was funded through the Canada Graduate Scholarships-Master's program (AZ), the Centre for Blood Research Graduate Award program (M-SS), the British Columbia Graduate Student Award program (M-SS), and the Canadian Institutes of Health Research grant (HOP-123801 to $\mathrm{HC})$.

Kauppila, T. E., Kauppila, J. H., and Larsson, N. (2017). Mammalian mitochondria and aging: an update. Cell Metab. 25, 57-71. doi: 10.1016/j.cmet.2016.09.017

Kennedy, S. R., Salk, J. J., Schmitt, M. W., and Loeb, L. A. (2013). Ultrasensitive sequencing reveals an age-related increase in somatic mitochondrial mutations that are inconsistent with oxidative damage. PLoS Genet. 9:e1003794. doi: 10.1371/journal.pgen.1003794

Kiyosawa, H., Suko, M., Okudaira, H., Murata, K., Miyamoto, T., Chung, M. H., et al. (1990). Cigarette smoking induces formation of 8-hydroxydeoxyguanosine, one of the oxidative DNA damages in human peripheral leukocytes. Free Radic. Res. Commun. 11, 23-27. doi: 10.3109/10715769009109664

Kreutzer, D. A., and Essigmann, J. M. (1998). Oxidized, deaminated cytosines are a source of $\mathrm{C} \rightarrow$ T transitions in vivo. Proc. Natl. Acad. Sci. U.S.A. 95, 3578-3582. doi: 10.1073/pnas.95.7.3578

Lindahl, T., and Andersson, A. (1972). Rate of chain breakage at apurinic sites in double-stranded deoxyribonucleic acid. Biochemistry 11, 3618-3623. doi: 10.1021/bi00769a019

Lindahl, T., and Karlstrom, O. (1973). Heat-induced depyrimidination of deoxyribonucleic acid in neutral solution. Biochemistry 12, 5151-5154. doi: 10.1021/bi00749a020

Lindahl, T., and Nyberg, B. (1974). Heat-induced deamination of cytosine residues in deoxyribonucleic acid. Biochemistry 13, 3405-3410. doi: 10.1021/bi00713a035

Loft, S., Vistisen, K., Ewertz, M., Tjønneland, A., Overvad, K., and Poulsen, H. E. (1992). Oxidative DNA damage estimated by 8-hydroxydeoxyguanosine excretion in humans: influence of smoking, gender and body mass index. Carcinogenesis 13, 2241-2247. doi: 10.1093/carcin/13.12.2241

Lohse, N., Hansen, A. E., Pedersen, G., Kronborg, G., Gerstoft, J., Sørensen, H. T., et al. (2007). Survival of persons with and without HIV infection in Denmark, 1995-2005. Ann. Intern. Med. 146, 87-95. doi: 10.7326/0003-4819-146-2-200701160-00003

Longley, M. J., Nguyen, D., Kunkel, T. A., and Copeland, W. C. (2001). The fidelity of human DNA polymerase gamma with and without exonucleolytic proofreading and the p55 accessory subunit. J. Biol. Chem. 276, 38555-38562. doi: 10.1074/jbc.M105230200

López-Otín, C., Galluzzi, L., Freije, J. M., Madeo, F., and Kroemer, G. (2016). Metabolic control of longevity. Cell 166, 802-821. doi: 10.1016/j.cell.2016.07.031

Matkarimov, B. T., and Saparbaev, M. K. (2020). "DNA repair and mutagenesis in vertebrate mitochondria: evidence for asymmetric DNA strand inheritance," in Mechanisms of Genome Protection and Repair, ed D. O. Zharkov (Cham: Springer), 77-100.

Merkwirth, C., Jovaisaite, V., Durieux, J., Matilainen, O., Jordan, S. D., Quiros, P. M., et al. (2016). Two conserved histone demethylases regulate mitochondrial stress-induced longevity. Cell 165, 1209-1223. doi: 10.1016/j.cell.2016.04.012

Michikawa, Y., Mazzucchelli, F., Bresolin, N., Scarlato, G., and Attardi, G. (1999). Aging-dependent large accumulation of point mutations in 
the human mtDNA control region for replication. Science 286, 774-779. doi: $10.1126 /$ science. 286.5440 .774

Miquel, J., Economos, A. C., Fleming, J., and Johnson, J. E. (1980). Mitochondrial role in cell aging. Exp. Gerontol. 15, 575-591. doi: 10.1016/0531-5565(80)90010-8

Palikaras, K., Lionaki, E., and Tavernarakis, N. (2015). Coordination of mitophagy and mitochondrial biogenesis during ageing in C. elegans. Nature 521, 525-528. doi: 10.1038/nature14300

Payne, B. A. I., Wilson, I. J., Hateley, C. A., Horvath, R., Santibanez-Koref, M., Samuels, D. C., et al. (2011). Mitochondrial aging is accelerated by antiretroviral therapy through the clonal expansion of mtDNA mutations. Nat. Genet. 43, 806-810. doi: 10.1038/ng.863

Scialò, F., Sriram, A., Fernández-Ayala, D., Gubina, N., Lõhmus, M., Nelson, G., et al. (2016). Mitochondrial ROS produced via reverse electron transport extend animal lifespan. Cell Metab. 23, 725-734. doi: 10.1016/j.cmet.2016.03.009

Spelbrink, J. N., Toivonen, J. M., Hakkaart, G. A., Kurkela, J. M., Cooper, H. M., Lehtinen, S. K., et al. (2000). In vivo functional analysis of the human mitochondrial DNA polymerase POLG expressed in cultured human cells. J. Biol. Chem. 275, 24818-24828. doi: 10.1074/jbc.M000559200

Trifunovic, A., Wredenberg, A., Falkenberg, M., Spelbrink, J. N., Rovio, A. T., Bruder, C. E., et al. (2004). Premature ageing in mice expressing defective mitochondrial DNA polymerase. Nature 429, 417-423. doi: $10.1038 /$ nature 02517
Yasui, M., Kanemaru, Y., Kamoshita, N., Suzuki, T., Arakawa, T., and Honma, M. (2014). Tracing the fates of site-specifically introduced DNA adducts in the human genome. DNA Repair. 15, 11-20. doi: 10.1016/j.dnarep.2014. 01.003

Ziada, A. S., Lu, M. Y., Ignas-Menzies, J., Paintsil, E., Li, M., Ogbuagu, O., et al. (2019). Mitochondrial DNA somatic mutation burden and heteroplasmy are associated with chronological age, smoking, and HIV infection. Aging Cell 18:e13018. doi: 10.1111/acel. 13018

Zsurka, G., Peeva, V., Kotlyar, A., and Kunz, W. S. (2018). Is there still any role for oxidative stress in mitochondrial DNA-dependent aging? Genes 9:175. doi: 10.3390/genes 9040175

Conflict of Interest: The authors declare that the research was conducted in the absence of any commercial or financial relationships that could be construed as a potential conflict of interest.

Copyright (c) 2020 Ziada, Smith and Côté. This is an open-access article distributed under the terms of the Creative Commons Attribution License (CC BY). The use, distribution or reproduction in other forums is permitted, provided the original author(s) and the copyright owner(s) are credited and that the original publication in this journal is cited, in accordance with accepted academic practice. No use, distribution or reproduction is permitted which does not comply with these terms. 